\title{
Transient pulmonary infiltrations in cystic fibrosis due to allergic aspergillosis
}

\author{
MARGARET MEARNS, WINIFRED YOUNG, AND JOHN BATTEN \\ From the Queen Elizabeth Hospital, Hackney, and the Brompton Hospital, London
}

In most patients with cystic fibrosis the morbidity and mortality are largely determined by the severity of the lung lesion. Staphylococcus pyogenes is usually found in the sputum and causes a chronic suppurative bronchiolitis and bronchitis with spread of the infection to adjacent alveoli. With more effective chemotherapy other organisms, such as Pseudomonas pyocyanea, are now being cultivated from the sputum with increasing frequency and may predominate. Certain fungal infections such as monilia, although feared, have not apparently occurred. Recently, however, two cases of cystic fibrosis have been encountered in which Aspergillus fumigatus was found in the sputum and in which the patients developed sensitivity to the fungus with pulmonary infiltration and eosinophilia.

\section{CASE REPORTS}

CASE 1 Born 2 June 1950. Although this boy had been suffering from progressive malnutrition and alimentary symptoms, the diagnosis of cystic fibrosis, based on absent duodenal trypsin, was not made until he was 2 years old in 1952. (This diagnosis was confirmed when the sweat was analysed in 1964 and showed a sodium concentration of $98 \mathrm{mEq} / 1$.: normal values are less than $60 \mathrm{mEq} / \mathrm{l}$.) He developed severe bronchitis at that time, and the chest radiograph showed patchy collapse, which recurred after a short course of antibiotics. With more intensive treatment improvement was sustained.

He remained well until 1958, with no evidence of lower respiratory infection and normal chest radiographs. However, he had frequent episodes of upper respiratory infection, and Staph. pyogenes was often present in pharyngeal swabs, for which long courses of antibiotics were prescribed.

In 1958 he developed hay fever, and the peripheral blood showed some eosinophilia, but this had also been recorded from time to time in previous years. He had an unproductive cough, which recurred in the summer of 1959 when he again had hay fever.

In April 1960 routine radiography showed a shadow at the right apex, although physical examination was negative. In July, when hay fever recurred, repeat films showed fresh opacities in both upper lobes. Clinically he was unwell with cough and marked wheeze, and Staph. pyogenes was again cultivated from the sputum. After treatment the radiological shadowing almost completely cleared (Fig. 1). He remained generally well, but had occasional short wheezy attacks, and during the winter of 1960-1 crepitations were heard from time to time at the right apex.

In July 1961 he expectorated blood-streaked sputum, and a week later he developed pleurisy, a pleural rub being heard over the right upper chest anteriorly. In August radiographs (Fig. 2) showed consolidation in the right upper lobe and left mid zone with enlarged hilar lymph nodes. He was given antibiotics, orally and by aerosol, and intensive physiotherapy. By October his general condition had improved and the chest radiograph showed clearing of the upper lobes. A productive cough, however, persisted. Staph. pyogenes continued to be isolated from his sputum, and antibiotics were given by aerosol and by mouth.

In January 1962 he developed generalized wheezing and persistent eosinophilia in the peripheral blood. The eosinophil count rose to $2,900 /$ c.mm. He was coughing more, had thick purulent sputum, and became dyspnoeic and cyanosed. He lost weight, and there was an increase in finger clubbing. Chest films in April 1962 (Fig. 3) showed new shadowing in the right lung and in the left mid-lung field, with enlarged hilar lymph nodes. Staph. pyogenes persisted in the sputum, but $\boldsymbol{A}$. fumigatus was also grown. In August he started to expectorate large plugs of sputum, and a dense shadow appeared in the right mid zone (Fig. 4), but his clinical condition gradually improved. He gained weight, the white count fell, and Staph. pyogenes was eliminated from the sputum; the chest radiograph started to clear some weeks later. By October 1962 the patient was fully active and he returned to school. By January 1963 he was said to cough very little and to produce sputum only after physiotherapy, but a few crepitations persisted at the right apex (Fig. 5). In June 1963 allergic aspergillosis was suspected and confirmed by finding a skin reaction positive to $A$. fumigatus antigen and precipitins in the blood.

Subsequently, further radiographs have shown transient shadows (e.g., Fig. 6), but abnormal shadows 
FIGS 1 To 7. Case 1. Serial P.A. chest radiographs from July 1960 to January 1964 show transient dense massive shadowing in both lungs with occasional hilar lymph node enlargement. There is residual shadowing in all zones.

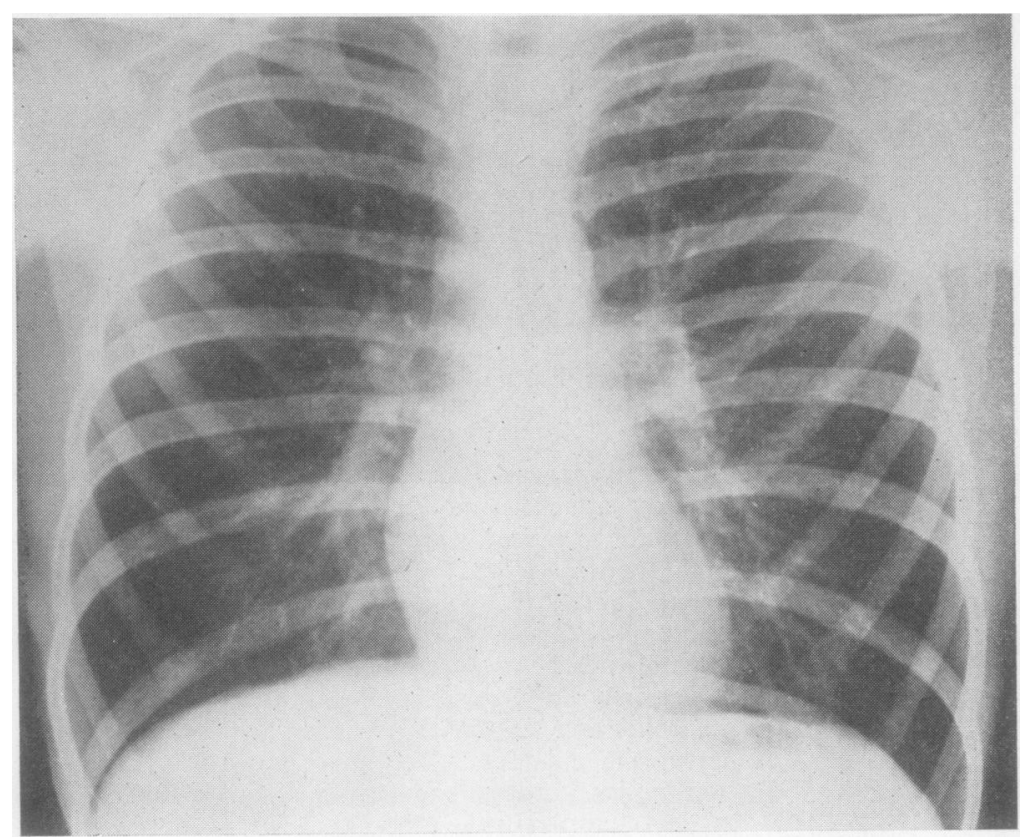

FIG. 1. July 1960.

FIG. 2. August 1961.

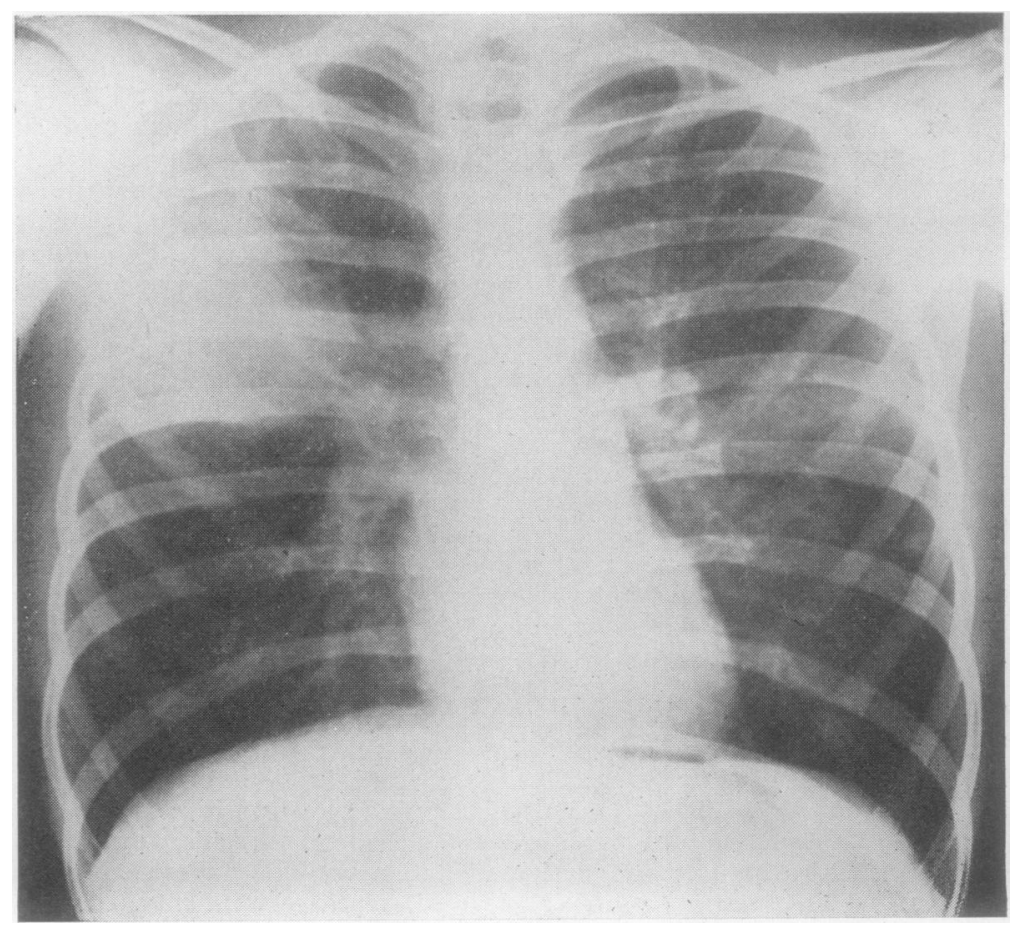


Transient pulmonary infiltrations in cystic fibrosis due to allergic aspergillosis

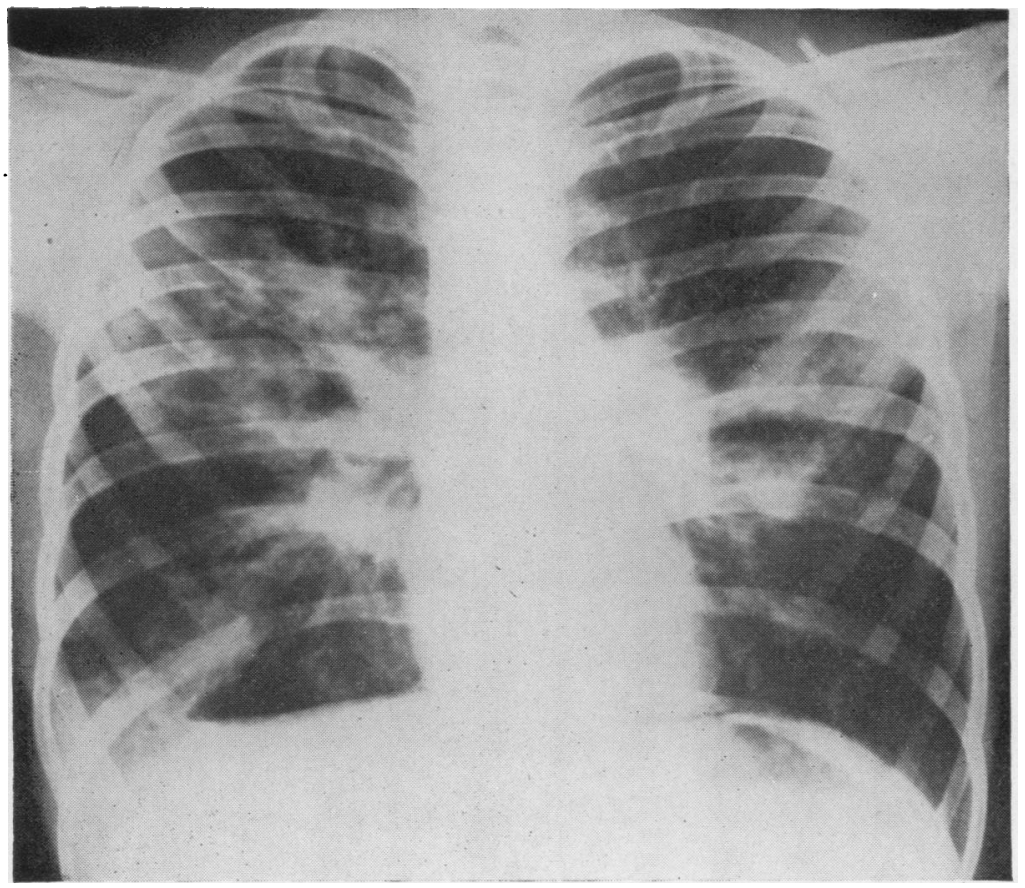

FIG. 3. April 1962. 


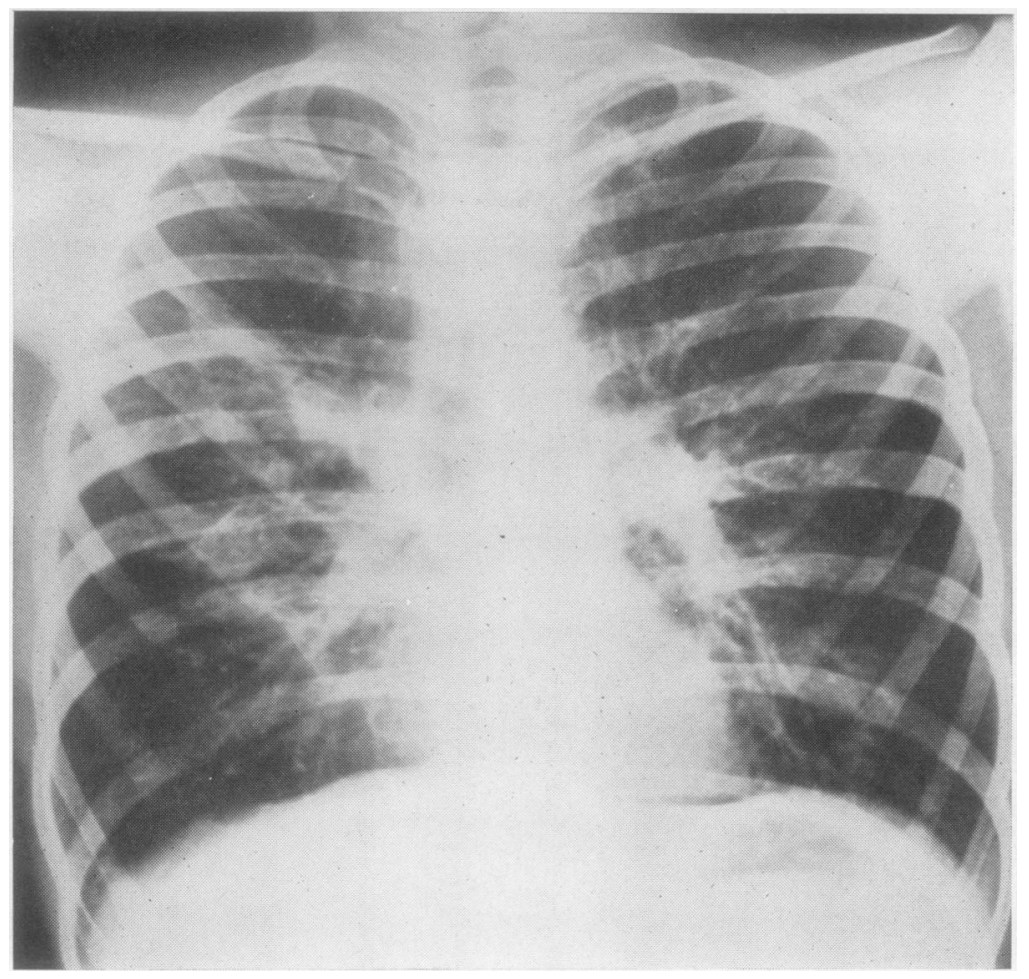

FIG. 5. January 1963.

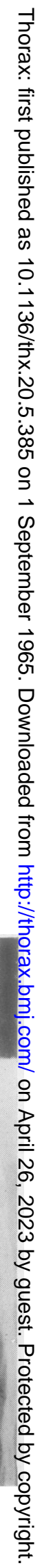




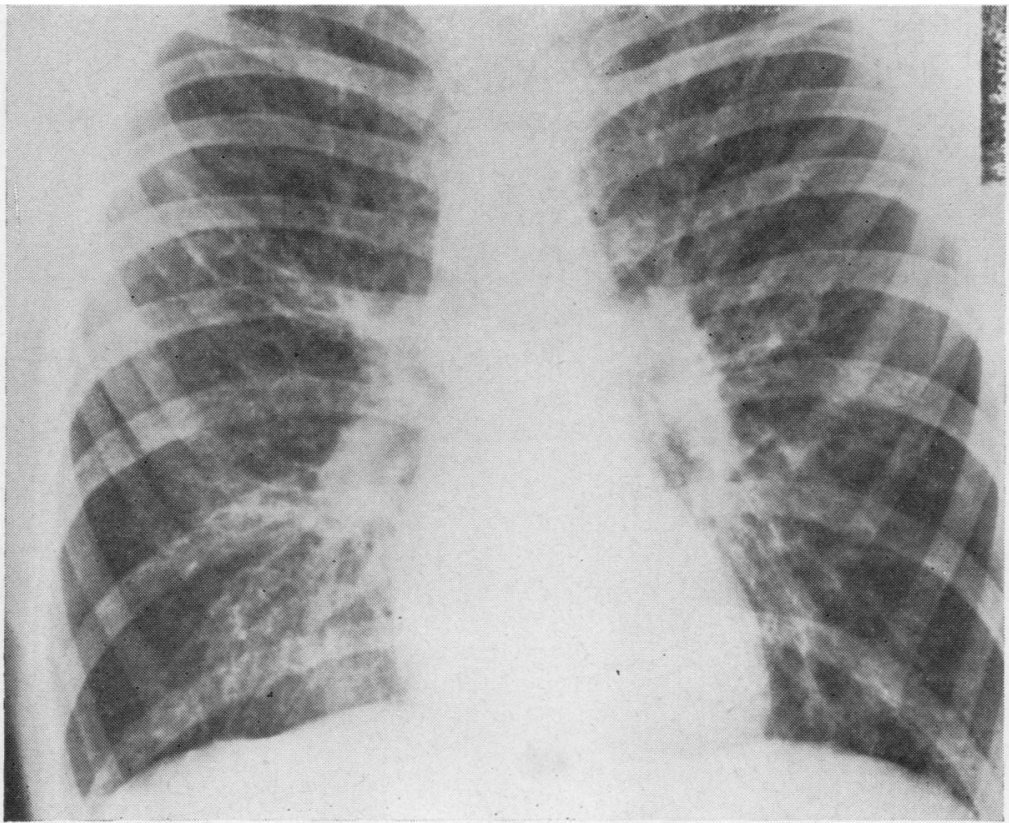

FIG. 7. January 1964.

FIGS 8 to 11. Case 2. Serial P.A. chest radiographs from September 1963 to August 1964. In addition to the persistent shadowing in the lingula, there are transient massive and dense shadows in the right lung field.

FIG. 8. September 1963.

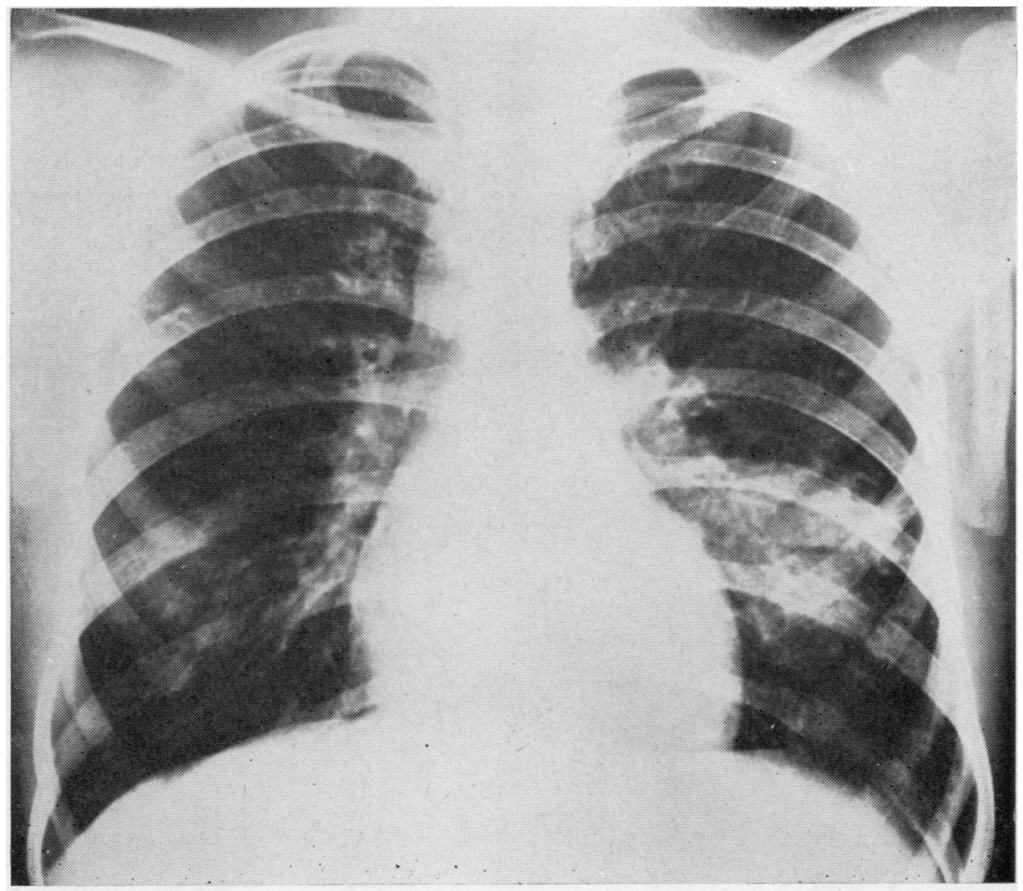




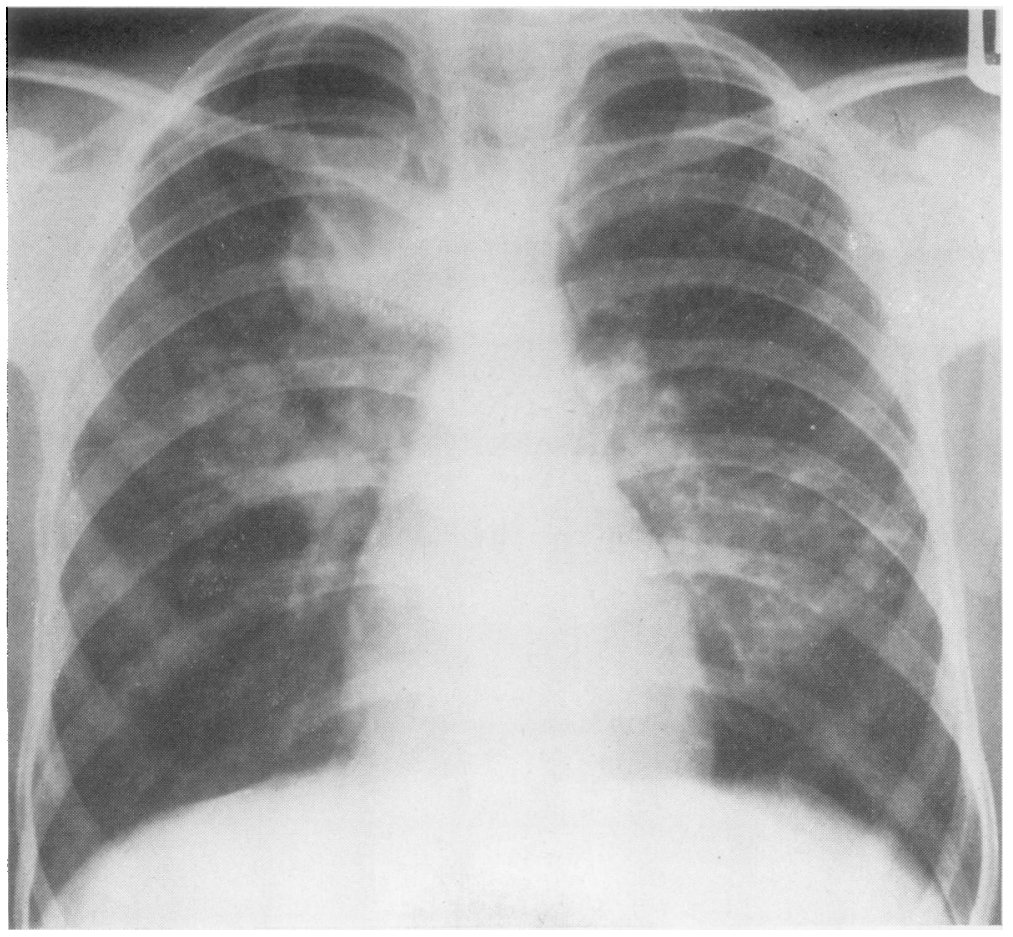

FIG. 9. February 1964.

FIG. 10. April 1964.

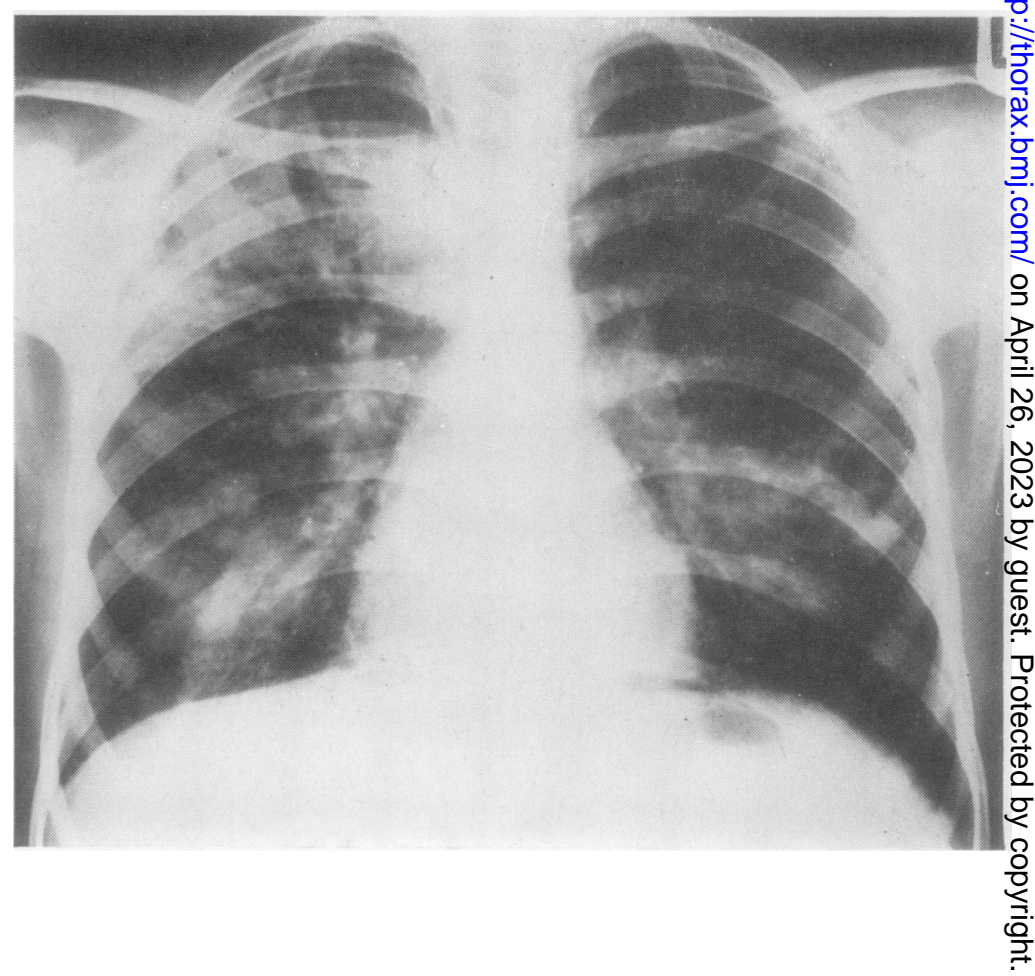




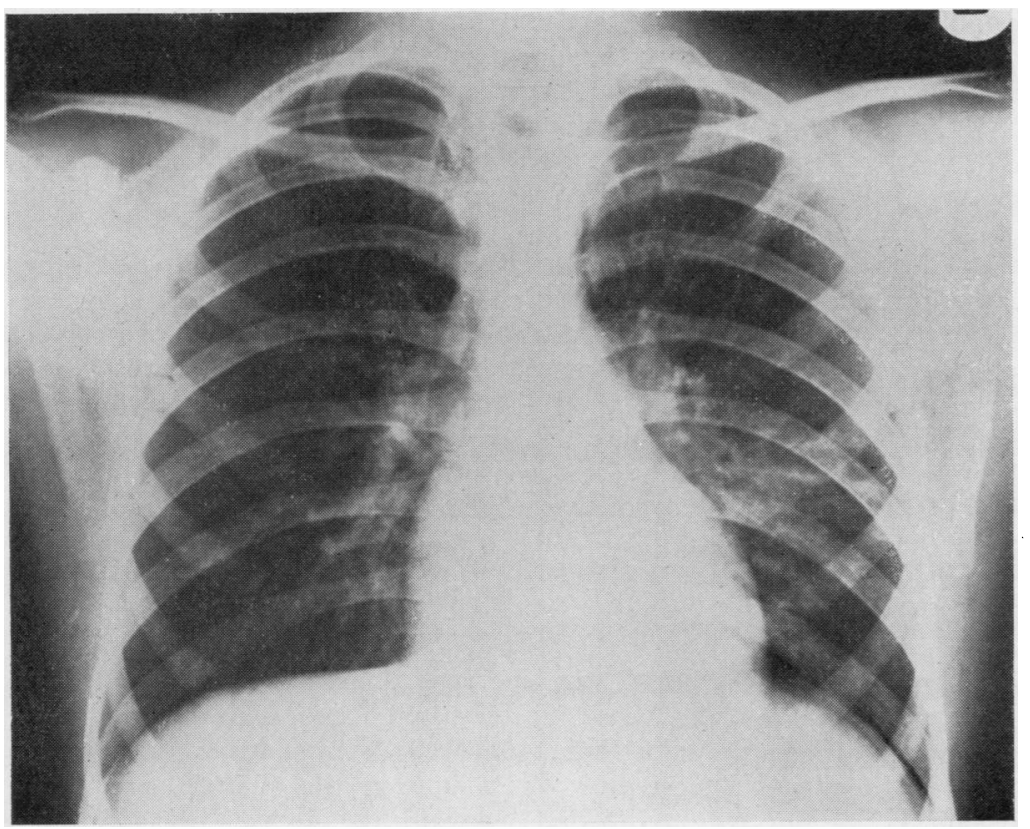

FIG. 11. August 1964.

now persist in all zones (Fig. 7). The eosinophil count has fallen to $800 / \mathrm{c} . \mathrm{mm}$. The sputum and throat swab remain clear of Staph. pyogenes. His general condition has improved, he gains weight, and is normally active for his age. He seldom coughs and only wheezes occasionally, but he produces variable amounts of purulent sputum on effort. The hay fever recurs each summer, but is less severe since desensitization. Antibiotics by aerosol were discontinued in May 1963 but have been continued orally.

CASE 2 Born 17 April 1953. Cystic fibrosis was diagnosed at 10 months of age in this girl, because of steatorrhoea and absence of trypsin in the duodenal contents. The sweat was analysed in 1963 , the sodium concentration being $145 \mathrm{mEq} / 1$. (normal $<60 \mathrm{mEq} / 1$.). Up to November 1962 she had had 11 admissions to hospital for chest infections, which were treated with a variety of antibiotics given for short periods.

In July 1962 the chest radiograph showed consolidation of the left upper lobe and disease in the lingula, and in October 1962 bronchoscopy was performed. Quantities of thick mucoid material were removed from the lingula, and a complete block in the lingula and apical lower segmental bronchi was demonstrated. Further radiographs showed no improvement until, after seven weeks' intensive treatment, the abnormal signs in the chest cleared and the film showed re-expansion of the lingula.

In September 1963, following a cold, crepitations were again heard in the lingular area, and the chest radiograph showed an opacity there (Fig. 8). This began to clear, and there was clinical improvement in response to intensive treatment. However, at the end of February 1964 the patient began to wheeze intermittently, and the sputum was occasionally streaked with blood. A radiograph at that time (Fig. 9) showed two unusually dense circular opacities in the right upper lobe, and there was a peripheral blood eosinophilia, the total count rising to $2,200 / \mathrm{c} . \mathrm{mm}$. Allergic aspergillosis was suspected. A skin test with Aspergillus antigen gave a positive immediate reaction. $A$. fumigatus was cultivated from the sputum and $A$. fumigatus precipitins were found in the blood.

The patient became generally unwell with dyspnoea, intermittent fever, and retention of infected secretions. In addition to the previous treatment she was given a 1:5,000 solution of brilliant green by aerosol three times daily.

A further chest radiograph in April showed fresh shadows in the right lung (Fig. 10). In May and June the films showed some clearing, the eosinophil count fell to $500 / \mathrm{c} . \mathrm{mm}$., and her general condition improved with a regular gain in weight.

In August 1964 the chest radiograph showed that the lingular lesion persisted, but there were only minimal changes in the right lung (Fig. 11). The child only coughs on effort and has little or no sputum. She is now fully active with good exercise tolerance.

\section{DISCUSSION}

We believe that these are the first two cases of cystic fibrosis to be described in which pulmonary infiltration with blood eosinophilia has developed due to infection with, and hypersensitivity to, the 
fungus $A$. fumigatus. This is established by the finding of the fungus in the sputum, precipitins in the blood, and skin hypersensitivity. The syndrome was first described by Hinson, Moon, and Plummer (1952), further clarified by Pepys, Riddell, Citron, Clayton, and Short (1959), and recently comprehensively reviewed by Campbell and Clayton (1964). The majority of cases described have had asthma: both our patients wheezed, but neither had severe asthma; one had hay fever. A family history of allergy is reported in about half the cases of allergic aspergillosis: one of our patient's brothers and his maternal grandfather had asthma, the other had no family predisposition.

Certain features are common to the previous clinical course of both these patients. Although each had suffered from episodes of pulmonary infection during early childhood, evidence of chronic infection with destruction of areas of the lung was delayed in the first child until 10 years of age, and in the second until 8 years of age. $A$. fumigatus was isolated from both of them two years later during episodes of pulmonary infection with retention of infected secretions for which intensive physiotherapy and antibiotics by aerosol and mouth were being given. Increasing susceptibility to pulmonary infection seemed to be associated with the development of allergic rhinitis in the first case, and wheezing in both.

The development of allergic aspergillosis may be a serious setback for these patients who may suffer repeated lung infiltration with irreversible bronchial damage. In this condition response to antifungal agents (e.g., nystatin, brilliant green, etc.) by any route or adrenocortical steroids has been poor. The true incidence of this complication in cystic fibrosis is not known, but these two are $\overrightarrow{0}$ among 100 or so patients attending one clinic. The incidence of significant Aspergillus or other fungal infection in the bronchial tree of patients with cystic fibrosis is unknown and is the subject of a co-operative study which has recently been initiated. With more effective chemotherapy and radical alteration in the bacterial flora of the respiratory tract, it is possible that fungal infection is becoming more prevalent in cystic fibrosis.

\section{SUMMARY}

Two patients with cystic fibrosis are described in $\frac{\mathbb{2}}{3}$ whom transient lung shadows were observed in $\stackrel{\mathbb{C}}{-}$ the chest radiograph associated with blood $\vec{\bullet}$ eosinophilia; at the same time Aspergillus or fumigatus was found in the sputum, precipitins to the fungus were found in the blood, and skin hypersensitivity was demonstrated.

\section{REFERENCES}

Campbell, M. J., and Clayton, Y. M. (1964). Bronchopulmonary aspergillosis. Amer. Rev. resp. Dis., 89, 186.

Hinson, K. F. W., Moon, A. J., and Plummer, N. S. (1952), Bronchopulmonary aspergillosis-a review and a report of eight new cases. Thorax, $7,317$.

Pepys, J., Riddell, R. W., Citron, K. M., Clayton, Y. M., and Short, E. I. (1959). Clinical and immunologic significance of A spergillus fumigatus in the sputum. Amer. Rev. resp. Dis., 80, 167. 